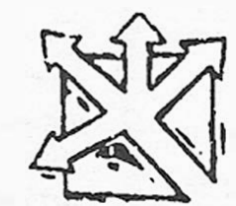

Educación Superior

\title{
CONFORMACIÓN DE UN EQUIPO DE INVESTIGADORES: UNA EXPERIENCIA CONCRETA.
}

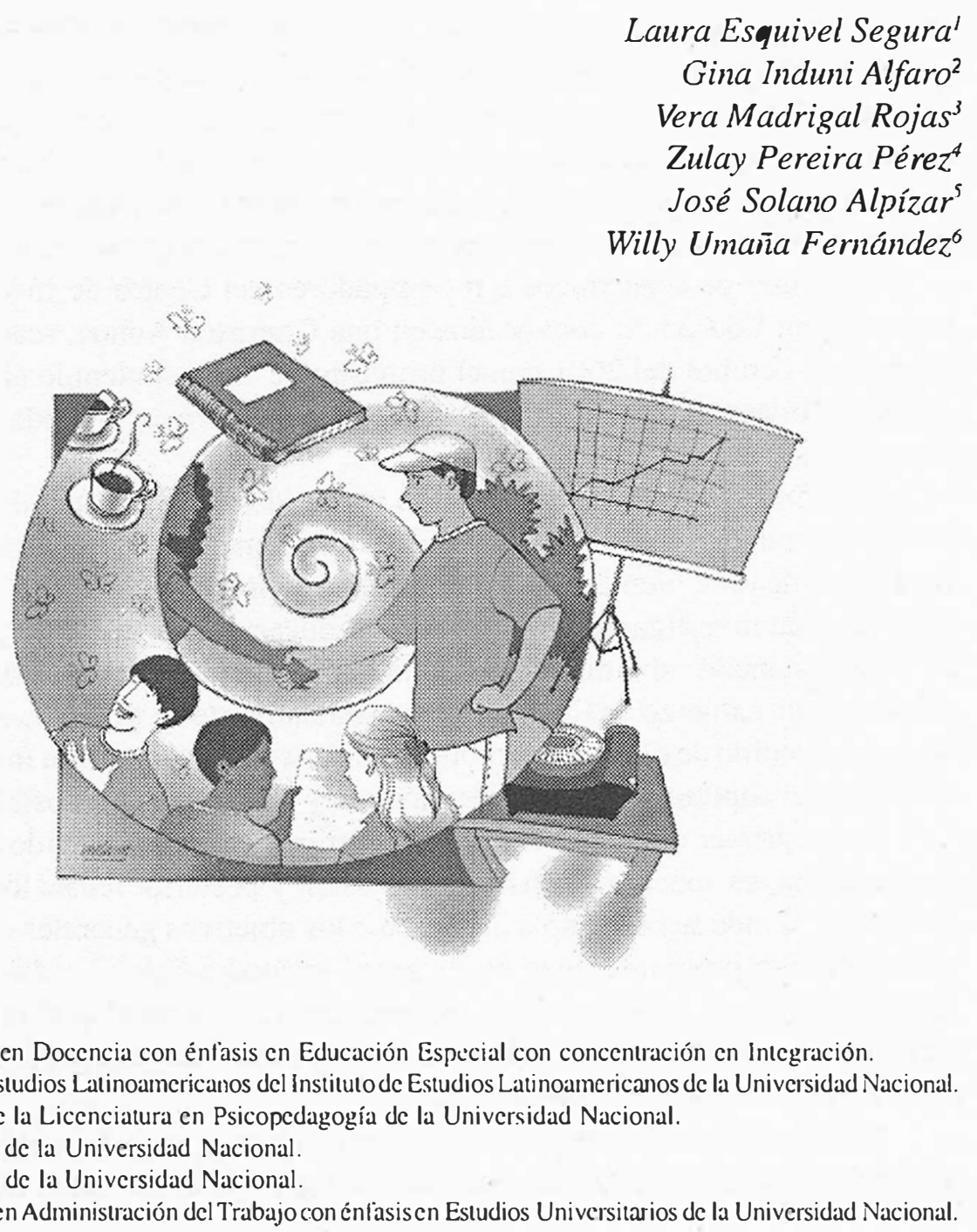

1 Licenciada en Docencia con énfasis en Educación Especial con concentración en Intcgración.

2 Máster en Estudios Latinoamericanos del Instituto de Estudios Latinoamericanos de la Universidad Nacional.

3 Egresada de la Licenciatura en Psicopedagogía de la Universidad Nacional.

4 Catedrática de la Universidad Nacional.

5 Catedrático de la Universidad Nacional.

o Licenciado en Administración del Trabajo con énfasisen Estudios Universitarios de la Universidad Nacional. 
Este es el primero. de dos artículos

En el Centro de Investigación y Docencia en Educación (CIDE) de la Universidad Nacionial. se han venido realizando un conjunto de esfuerzos acudémices y de gestión con el propur. sito de posicionar al Centru cumo uno de los principales reforentes educativess del país y del íiea centroamericinna.

Dentro de Éslos, el potenciamiento de la investigación hajo lia cunformación de un Programa de lıvestigación acenas de la experiencia vivida por un grupo de académicos del CIDE. en la conformacioun de un ernipo de investigudores. En el primer arliculo se señalum no sólo los origenes de la ielea, sino rambicin las erapas vividas. las es. rrurcgias urilizadas para su estimulacionn, asi como algunas sugerencius ene podrian ser b'?eficiosas para orros grupos epue se enfren. ren a sareas similares. El segundo arriculo precisa el abordaje y ln estrasegia merodolosica adopuadu por el eanipo, asi cumo el proceso consirucrivo para llegar a ese acercamicnto mesodulógico.

Educativa (P. I. E.) que reúna a investigadores de todas las Unidades Académicas a fin de consolidan equipos interunidades $e$ interdisciplinarios, se ha constituido en un ver. dadero logio, en momentos en que la educación costarricense requiere de los mejores esfuerzos para dar respuesta a las di versas problemáticas en que se encuentra inmersa.

Un grupo de académicos e investigadores del Centro de Investigación y Docencia en Educación convocados en una Comisión Adhoc, sesionó a partir del mes de octubre del 2001 con el propósito de darle contenido al futuro Programa de Investigación Educativa del Centro y al proyecto madre con el que iniciaría su queliacer dicho programa.

Esta Comisión estuvo conformada por académicos y académicas del Centro (2002), quienes impulsaron la idea del Programa de Investigación Educativa. Dicha idea fue bien acogida y respaldada ampliamente por las autoridades del Centro de Investigación y Docencia en Educación, por su Consejo Académico y las instancias administrativas de la Universidad Nacional. Este programa constituye un esfuerzo de Centro y no una iniciativa de un grupo de académicos, bajo el entendido de que la sostenibilidad de una actividad de esta índole requiere de todo el soporte académico, económico y administrativo posible.

En un primer momento el equipo encargado de dar contenido a la idea de un programa, se abocó al análisis, la discusión y posterior redacción del borrador inicial, donde se contempla claramente los objetivos generales que se persigue con dicho programa. Este busca ser el encuadre físico-académico de una serie de proyectos, subproyectos y actividades que nutran el quehacer del Centro de Investigación y Docencia en Educación y posibiliten una proyección mayor en el ámbito nacional e intemacional.

Unido a la tarea de pensar un Programa de Investigación Educativa, nombrado como "Educación y Desaitollo", estuvo ligado la elaboración de un primer 
First of mo articles describing ihe ex. perience shared by a group) of CIDE (Silhool of Educarion an UNA. Cosia Rica) scholurs in conforming a sean of reseurchers. The first points out wos only how the ided wets originated but atso the differens srages it "uent ihrough and the stralegie's used to slimulased it. In addition. some suggessions are gine'n so that fun rure researches may. ben. e fin from them when facing similar usesk. The secenter arricle defores the methodological sirute.s. adopled by the leam and ilse' comstrucivic process then was followed to rench such methodological approuch.

"proyecto -madre", que posibilitara cl despegue del Programa. Estc proyecto presentado en su borrador final ante las instancias universitarias correspondientes en cl año 2002. fuc aprobado y' se le concedicron tres ticmpos complctos para quc inicialia labores en el tercer trimestre del año 2003.

El documento borrador de dicho proyecto. tiaulado "Perfiles. di. nímicas y desalios de lis celucación costarricense". contempla una descripción del cstado en que se encuentra la cducación costarricense y' sc plantca una scric de lincamicntos y actividades que sirvicron de insumo para el cquipo del Programa de Investigación Educativa en su fasc de conformación y propucsta de trabajo futuro.

\section{Introducción}

"Intcgrarse en un grupo cs mucho más que compartir algunos momentos, algunas actividades, y algunas idcas. Integrarse es recorrer $\mathrm{cl}$ camino del encuentro haciendo propias las inquietudes, los descos y la búsqueda de todos los que conforman cl grupo" (Autor desconocido, citado por Esquivel, 2002 ).

En cualquier grupo de personas que se reúnan con el propósito de conformarse en un equipo para realizar una labor específica, perseguir objecivos concretos, diseñar proyectos y ejecutarlos, o en términos generales buscar una misma meta, se vive un proceso determinado que pocas veces suele transcribirse o registrarse.

No obstante, el haccrlo puede servir como clemento de guía para futuras cxperiencias, o tambićn para visualizar de una manera más precisa el crecimiento vivido y las estratcgias scguidas para favorecer la conformación del cquipo.

Es por cllo, que dentro del Programa de Investigación Educativa del Centro de Investigación y Docencia en Educación sc ha pensado en registrar de una mancra coloquial las diferentes etapas que ha vivido el grupo de profesionales que se han rcunido para ejecutar el primer proyecto adscrito al Programa, sicndo esta la primera experiencia que se tendrá y sabiendo que en cl futuro se irán a 
vivir diferentes elapals de conformakión de equipos. lals cuales bien podrian verse facilitadals al contar con una descripción de los aspectos o siluaciones que han debido enfientarse en este primer periodo de cjecución del Programal.

Es biljo csil optical que se hal optiado por halcer un recuento de estil experiencia, que como se hal dicho es mis anecdóical que técnical, no obstante. sc considera que no por elío. es de menor valer. Se invital al lector a analizalr las elapas que esic grupo de profesionalcs ha vivido $y^{\prime}$ a sacar de cllas cl mejor provecho posible, de modo que sus propias cxperiencias al incorporarse a grupos o equipos paral trabajo se vein favorecidas.

\section{Etapas vividas}

\section{Conocimiento de las personas participantes}

Se considera imporlante registrar el momento en que los /ats alciadémicos / as se reúnen. sus realceiones, la dinimicias del grupo, o cn otros licrminos historizalr su constilución, porque se parte del supucsto de que un grupo no se constiluge exclusivamente por la presencial de sus miembros y la sumaltoria de sus ciariaclerísticals, ni por $\mathrm{cl}$ hecho de que se encuentren personals convocialals en un cspilcio físico delerminado. Se asume entonces que un grupo estí realmente conslituido por unal red de relaciones y vínculos entre personals, que buscian, compiaten o trabajan en función de objctivos comuncs y por tanto, desalurollan un sentido de pertenencial y de apropiación.

Esta hal sido la primeral etapal en la cual los profesionales asignados al Programa de Invesligación Educalivil del CIDE, se conocen, se relacionaln muchos de ellos por primeral vez, es interesintc señalar que los participantes, son de diferentes especialidiades, en el calso concreto del relato de estal experiencial es importante señalar que se tralla de un grupo intergeneracional e interdisciplinario:

En cuanto al género, el grupo está conformado inicialmente por tres varones y cuatro mujeres. Uno de ellos fue un estudiante avanzado de la ciarera de Orientación, no obstante en las primeras reuniones manifiesta que val al priorizar sus estudios, razón por la cual agradece la oportunidad de participar como parte del grupo de trabajo, pero se retirará para terminar su carrera. En el grupo se manifiesla que habría sido muy importante contar con la presencia esludiantil en este trabajo, no obstante, se ha de asumir la no participación por decisión propia del esludiante.

Los otros tres participantes son profesionales con mayor experiencia en el campo de la investigación, tres profesionales que se iniciaban formalmentc en investigación. Dentro de las especialidades de los participantes están: Historia, Educición; Psicopedagogía y Educación Prcescolar; Psicología, Investigación 
y Dercchos Humanos: P'sicopedanogía, Música y Educación Especial; Planificación. Administración: Estudios Lattinoamcricanos y Psicología, y un cstudiantc de nivel alvanzado de la calrrera de Orientación.

Tienen en comin la experiencia docente, alunque en diferentes niveles y con difercntes poblaciones. lo cual les ha permitido construir una visión en conjunto del sistema cducativo, que se verí reflejada en el trabajo elaborado. Comparten un fuerte arraigo con la Universidad Nacional, pucs en su mayoría son estudiantes egresidos y/o docentes en ejcrcicio de dicha institución.

Otro hecho clave cs que la brecha generacional, que usualmente distancia a las personas, en esta ocisión ha funcionado como un reflejo de difcrentes perspectivas, lo cual ha enriquecido el trabajo grupal.

\section{Planteamiento de expectativas}

En las primeras reuniones, el grupo de académicos y cl estudiante, se encuentra con un planteamicnto del programa y del proyecto, producido por los gestores de la idea y reacciona de forma variada ante su revisión. En términos generales se hicieron observaciones en torno al grado en que el grupo participantc podría modificar la forma y el contenido de algunos elementos del discño original.

\section{Temores de los participantes}

Entre los patticipantes se evidenciaron temores o conflictos específicos, la mayoría de cllos referidos a los cimpos de especialidad y a las afinidades con determinados modos de ver cl mundo, la cducación y de opciones de abordaje de los problcmas de investigación, compatibles con sus vivencias.y opciones de investigación. Es así como surgen confrontaciones referidas a la afinidad o no con los objetivos del programa y del proyecto, lo cual es a criterio de algunos de los participantes un elemento a considerar dado que es difícil tener claridad y motivación hacia un trabajo grupil con el cual no se tenga compatibilidad, este fue un, aspecto que afloró en el grupo de mancra reiterada y que en varias ocasiones requirió de su análisis como parte de las sesiones de trabajo.

Inicialmentc podría señalarse que los dos aspectos antagónicos que el grupo vivió con mayor fucrıa y generaron mayor reflcxión fueron aqucllos referidos a los planteamientos del paradigma positivista y el paradigma naturalisti.

Micntras que algunos de los miembros del grupo pretcndían enfatizar en resultados (logros y alcances) del sistema cducativo formal costarricensc y en lat cxplicación del fenómeno educativo mediante la identificación de sus causas yf 
o factores determinantcs, basándose fundamentalmentc en información existente, mostrando con ello una inclinación eminentemente cuantitativa, centrada en la atención de aquellos aspectos observables y susceptibles de cuantificar y cuyos datos se analizarían con fundamento en la cstadistica.

Otros de los micmbros del grupo, por el contrario, propiciaban la utilización de técnicas cualitativas, buscando mediante la descripción proporcionada por las propias palabras de la personas involucradas, comprender c interprctar, dentro de su propia perspectiva, aquellos aspectos del fenómeno educativo considerados relevantes para dar una visión holística y lo para conocer cierta parte de la realidad para la que no se tiene información ni resultados disponibles. Por lo tanto, más interesados en las particularidades del contcxto en el que ocurren los hechos, en el cómo se llevan a cabo los procesos que median en el fenómeno educativo y en el cómo lo perciben los involucrados directos, enfatizando los aspectos más humanistas.

Desde las perspectivas anteriores, el grupo coincide con los planteamientos de Fabra y Doménech (2001), cn el sentido de que la rcalidad social para ser realmente comprendida no puede circunscribirsc cxclusivamente a la cuantificacion, sino que ha de abordar los significados y símbolos, lo cual solo es posible a partir del análisis de los rclatos de las personas implicadas.

Hubo también, señalamientos en cl scntido de que no era posible tener previamente establecidos en un programa y/o proyecto, los instrumentos u objetivos que respondian necesariamentc a un tipo de paradigma que quizá no cra del todo compartido por quienes harían el estudio. De igual manera se scñalaba que, quienes plantcaban opcioncs de caráctcr naturalista cacrían en el riesgo de que sus planteamicntos tampoco fueran aceptados por otros micmbros del grupo. Es quizá cste aspecto el que mayormente consumio horas de trabajo y discusion en cl grupo.

Se planteb, además, de qué manera un Programa de Investigación Educativa a nivel del Centro de Investigación y Docencia en Educacion, podría circunscribirsc a una opción epistemologica y cerrar paso a otro tipo de propucstas, o cn su defecto, de qué manera plantear las cosas que permiticran la complementaricdad de cstratcgias de trabajo y una mejor vision del objeto de cstudio del programa. El grupo sc fuc inclinando hacia esta segunda opción de una búsqueda de complementariedad y es a partir de ello que se aboca a la búsqueda cspecífica de matcrialcs de lectura que pudicran dar claridad sobre cse aspecto. 


\section{Estrategias de trabajo para promover un pensamiento compartido}

Una vez clarificado que el grupo de participantes, antes de diseñar el tipo de proyecto a realizar, descaba tener claridad sobre diferentes aspectos epistemológicos, metodológicos y lécnicos, se asigna a dos miembros del mis. mo, seleccionar alguna temática y buscar literatura específica para ser Icída y comentada por todos los participantes.

Es asi como se hacen dos scsiones de trabajo que versaron sobre paradigmas y sobre técnicas de recolección y análisis documental. Todos los miembros del grupo hicieron las lecturas correspondientes y en la siguiente sesión de trabajo, los encargados dirigieron la sesión haciendo un resumen y explicación de los aspectos medulares sobre la temática asignada, en la cual hubo amplia participación del resto de compañeros. De nucvo afloran en eslas sesiones los temores antes scñalados y se van proponiendo diferentes opciones de trabajo que permilirían ir avanzando en el diseño del proyecto a la ve\% que aportarán información que permitirá la clarificación teórica de los participantes.

\section{Búsqueda de ideales y metas comunes}

Se adoptó como rutina la estraltegia de trabajo que combina el csludio individual y la retroalimentación grupal. Y con el liempo esto fortaleció la comunicación de los participantes del grupo.

A raíz del trabajo de búsqueda bibliográfica en el cual participaron todos los /as micmbros del grupo, del análisis de la misma, se fueron dando sesiones de trabajo en las cuales iban decidiéndose algunos aspectos a abordar en un posible proyecto discñado conjuntamente, es así como se recopilan esas ideas, propuestas y sugerencias específicas, las cuales van quedando transcrilas en crónicas por scsión.

En dichas crónicas no solo se transcriben ese tipo de sugerencias, sino que lambién sc recopilan aquellos aspectos que van surgiendo como intereses específicos de los participantes, pero que a futuro podrían constituirse en posibles árcas de invesligación o en posibles estrategias de trabajo.

Esila estraltegia de recopilación y valoración de los aportes de caada uno de los participantes se fue constituyendo en una forma de dar confianzalal grupo y fuc permitiendo mayor espontancidad en las participaciones, más apertura para plantcar propucstas, aunque éstas no fucran posibles de cumplir o conscguir a corto o mediano plazo y fucron así surgiendo diferentes ideas que a futuro se espera que den gran fortalc zal al Programa de Investigación. 
A partir de estas acciones, empezaron a surgir de manera consensual algunas ideas que se volcaron en matrices de trabajo y en las cuales se consignaban categorias posibles de analizar en un proyecto específico y ya con este apoyo cl grupo empezó a haccr propuestas que poco a poco iban siendo incorporadas por los participantes.

Dado que se daban ya algunas propuestas que eran compartidas mayoritariamente, sc visitaron cspecialistas para realizar entrevistas que pudicran dar luz en algunos trechos del camino. Y se optó también como estratcgia la búsqueda de matcriales, que por su valor histórico y por la claridad epistcmológica dc los mismos, pudieran constituirse en un material estable en el programa y al cual sc pudiera recurrir de manera permancnte y cfcctiva.

Para esta iniciativa, se buscó el apoyo de la Decanatura del CIDE, que brindó cl aporte para quc en el grupo se pudiera contar con dicho material y al día de hoy, 4 mescs después, se cuenta ya con valiosos matcrialcs que a futuro podrán scr de utilidad para otros profesionales del Centro. Dentro del apoyo con que sc ha contado es disponer de una sala de trabajo, en la que se acondicionaron escritorios que permiten la pcrsonalización del trabajo, apoyo tccnológico que aún requiere de su total instalación, pero que el grupo rcconoce que scrá de gran ayuda para cl trabajo que se debe realizar.

Contar con un espacio de trabajo, que pucde ser tanto de carácter individual como grupal, se constituye en un apoyo importante no solo a nivcl académico, sino también afectivo. El poder compartir un espacio físico, el velar por dicho espacio y el equipo, el compartir horas de trabajo, de discusión, de interacción humana, entre otros aspectos, permite fortalecer las relaciones intcrpersonales y no solo eso, sino que facilita un proceso de identidad como miembro dc un Programa de Investigación Educativa que a larga scrá sumamente valioso en la consccución de los objetivos propuestos.

No sc puede dejar de lado la hora del café, bien conocida por los costarricenses como un cspacio, dentro de la cotidianidad, para cl descanso y la expansión, pcro también una forma de continuar la discusión en un escenario informal como una soda, que por su naturaleza da un matiz de confianza a la conversación. Este espacio, aunque corto, fue utilizado de manera espontánea para continuar cl análisis de las temáticas abordadas cn la sesión de cse día.

\section{Paso de un grupo a equipo de investigadores}

Las estrategias antes descritas, y el espacio de trabajo del cual se dispone, fucron generando en el grupo una sensación de estabilidad emocional, de respeto por las ideas y especialidades de los otros, de reconocimiento del valor que 


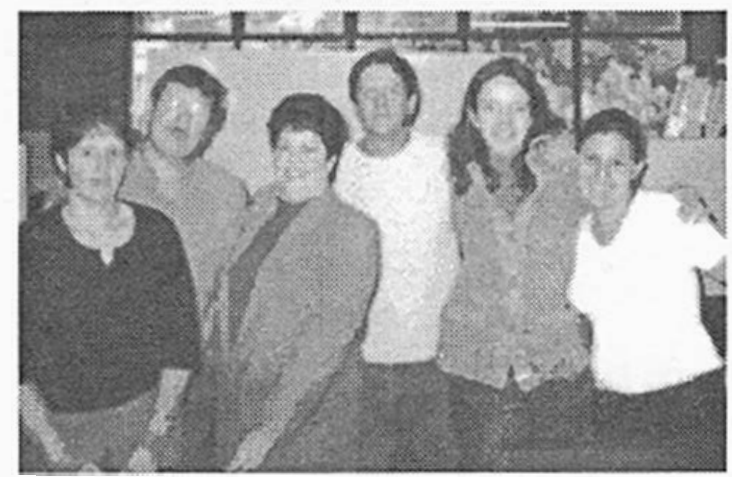

Equipo de Investigadores esıá conformado por Vera Madrigal, Willy Umaña. Zulay Percisa. Jose Solano. Gina Induni y Laura Esquivel. cada cual puede aportar y aporta al resto así como de un deseo explícito y compartido por la búsqueda de objetivos comunes.

Ahora se comparte la motivación por concretar ideas y utilizar aquello que se ha recopilado en las matrices de trabajo y en las crónicas. Ha sido posible ya diseñar una primera propuesta para el primer proyecto de investigación del programa, lo cual ha llevado a que el equipo concretice la búsqueda de objetivos de investigación y técnicas de recopilación y análisis. Ese proceso se ha logrado de manera compartida, consensual, lo cual ha permitido el paso de un gnupo de personas a lo que hoy puede visualizarse como el equipo de investigadores.

Las sesiones de trabajo se han tomado más dinámicas, más fáciles de realizar, sin que ello implique que no hay discusión, por el contrario, ahora ese compartir académico es mucho más rico, hay discusiones de fondo en aspectos epistemológicos y metodológicos, pero los mismos se asumen sin temor, con una claridad que no se tenía al inicio. La construcción compartida a un acercamiento epistémicometodológico ha facilitado el que de los debates iniciales se pase a un quehacer matizado por una mayor motivación para con el trabajo a realizar. En este sentido el equipo ha experimentado lo que bien apunta Aguilar (2003, p. 68) al señalar que:

"Los mejores equipos, posiblemente todos los equipos que sobreviven como equipos efectivos y afectivos, suelen tener conflictos internos entre sus miembros, sobre todo el principio. Si se impide que salga a relucir el conflicto y por tanto, que sea tratado adecuadamente, entonces ese equipo no llegará nunca a funcionar al máximo de sus posibilidades. Pero cuando los miembros del equipo afrontan el conflicto y lo resuelven entre ellos, entonces forman un equipo al cual cada uno le gustaría permanecer. Una tensión manejable es necesaria a la dinámica del diálogo, la cual supone: tesis, antítesis y síntesis, es decir, debate y consenso en lugar de monólogo y sumisa obediencia".

Al finalizarel año 2003, tres meses después de la primera sesión de presentación de los participantes, es posible señalar que se había logrado ya la conformación del 
equipo de investigadores y el diseño general del proyecto de investigación en cuanto a objetivos, estrategias de trabajo, organización interna del equipo y propuesta metodológica que regirá los diferentes proycctos que a futuro se adscriban al Programa de Investigación Educativa del Centro de Investigación y Docencia en Educación.

\section{Conclusiones}

La exposición que se ha hecho de la experiencia vivida por el grupo de profesionales asignados al Programa de Investigación Educativa del Centro de Investigación y Docencia en Educación y más concretamente al proyecto Perfiles, dinámicas y desafíos de la educación costarricense, hasta lograr conformarse como equipo de investigadores, es considerada de sumo valor por quienes la han vivido, pues significa clarificarse las etapas afrontadas, las estrategias utilizadas y el crecimiento que se ha logrado.

Mucho de lo vivido se ha ido planteando de manera espontánea, de un modo más informal que formal. No obstante, ha sido justamente esa búsqueda de una adecuada relación humana, donde se favorezca el crecimiento individual, el respeto por el pensamiento de la otra persona, la búsqueda de consenso para aspectos considerados medulares en el Programa, el aprovechar las especialidades y conocimientos de los diferentes participantes, ese respeto individual pero siempre matizado por la búsqueda de un fortalecimiento grupal, lo que a criterio de los participantes ha permitido el contar actualmente con un equipo de investigadores deseosos de sacar adelante este primer producto del Programa y abrir un camino para futuros grupos de investigadores del Centro de Investigación y Docencia en Educación, que con su trabajo investigativo promuevan el conocimiento, crecimiento y afronten los retos en el campo educativo.

\section{Sugerencias}

Una vez relatada la experienciavivida en la conformación del equipo de investigadores del Programa de Investigación Educativa del Centro de Investigación y Docencia en Educación, (P. I. E. - CIDE), es conveniente señalar a manera de sugerencia, algunos aspectos que se considera han de facilitar la constitución de cualquier equipo de investigadores /as, asi por ejemplo es válido señalar algunos elementos en función de las etapas vividas por el grupo antes mencionado:

1. Conocimiento de las personas participantes: en esta fase, es importante saber cuáles son las caracteristicas no solo profesionales, sino tambien de personalidad, de las personas que constituyen el grupo. Es también valioso rescatar y aclarar que independientemente de los años de experiencia, del 
recorrido de cada cual en el campo de la investigación, el equipo que se constituya tendrá sus propias características, su propio crecimiento y las ventajas que existan en algunos de los miembros a nivel de experiencia se podrán utilizar para fortalecerlos a todos y no para generar diferencias.

2. Planteamiento de expectativas: es valioso que cada persona participante tenga claridad acerca de las expectativas que le genera el haber sido asignado al programa o proyecto específico, ello permitirá que los compañeros y compañeras de grupo tengan claro la visión de cada uno y las metas u objetivos que a título personal poseen, ello ayuda a que todos comprendan mejor las intervenciones y planteamientos, tanto teóricos como metodológicos, que a futuro se irán presentando.

3. Temores de los participantes. En cualquier grupo de personas hay experimentación de temores, algunos de ellos suelen estar referidos al ámbito personal, pero otros pueden ser fácilmente ubicados en el campo estrictamente profesional o de la labor a realizar. Es en este último aspecto al que va dirigida la sugerencia de promover el ambiente de trabajo necesario, a fin de que dichos temores afloren sin estar asociados a señalamientos o desvalorizaciones por parte de los miembros del gnupo. Este aspecto que podría considerarse superlluo, es realmente imporante, porque su verbalización y comprensión serán la clave para un trabajo claro, de respeto y de búsqueda de altemativas para la incorporación activa de todos los participantes.

4. Estrategias de trabajo para promover un pensamiento compartido. Si las etapas anteriores se van llevando de manera apropiada, caracterizadas siempre, por una adecuada relación humana, por el respeto personal y profesional de cada uno de los participantes, es posible en este momento, aprovechar los conocimientos o habilidades de las diferentes personas y ponerlos a disposición del grupo en general. El recurrir a lecturas sobre temáticas específicas, al análisis de aquellos aspectos del programa o proyecto que han generado mayor disconformidad o preocupación, apoyarse en las opiniones de los planteamientos de los diferentes participantes ayudará a ir fortaleciendo al grupo y promoverá el paso a la siguiente etapa.

5. Búsqueda de metas e ideales comunes. Los puntos antes mencionados como sugerencias por etapas, permiten a este nivel empezar a identificar posibles metas o ideales comunes que irán sentando las bases para el diseño del programa o proyecto a desarrollar y como sugerencias puede recurrirse al uso de matrices que recopilen los planteamientos generales y compartidos en función de posibles objetivos, estrategias de trabajo, instrumentos u otros que promoverán a futuro el logro de las acciones que se diseñen. 
6. Paso de un grupo a un equipo de investigadores. En este momento es posible ya pensar de una manera más coordinada, los aspectos que pueden señalarse como facilitadores del paso de un grupo a un equipo de investigadores, son entre otros, el poder contar con un espacio físico que permita a los participantes reunirse e irformándose un sentido de pertenencia, buscar recursos quc faciliten el trabajo de todos, el análisis de materiales que se van identificando como básicos para el trabajo a realizar. En fin, son todas las etapas descritas en la experiencia vivida, las que deben irse atendiendo a su debido momento, pero en la medida en que estas sean facilitadas con las sugerencias indicadas, es posible sobrellevarlas de mejor manera y no verlas como aspectos que obstaculizan la conformación de los equipos de in vestigadores.

\section{Referencias}

Acta. (2001 ). Comision ad hoc. Heredia: CIDE.

Aguilar, R. (2003). Simpatímetro: de aprendiz a pensador - El peso de la inteligencia emocional-. III ensayo. Heredia: Defensoria del Estudiante, Universidad Nacional.

Esquivel, L. (2002). Propuesta para efectuar un taller de sensibilización aue promueva actitudes positivas hacia las personas con discapacidad y/o necesidudes educativas especiales, dirigido a docentes del sistema educativo regular. Memoria del Curso Especializado Adecuación Curricular II. San José: Universidad Estatal a Distancia.

Fabra, M. y Doménech, M. (2001). Hablar y escuchar: Relatos de profesor@s y estudiant@s. Barcelona: Paidós. 